\title{
Comparison of spectroscopically measured tissue alcohol concentration to blood and breath alcohol measurements
}

\author{
Trent D. Ridder \\ Benjamin J. Ver Steeg \\ Bentley D. Laaksonen \\ TruTouch Technologies, Inc. \\ 800 Bradbury South East, Suite 219 \\ Albuquerque, New Mexico 87106
}

\begin{abstract}
Alcohol testing is an expanding area of interest due to the impacts of alcohol abuse that extend well beyond drunk driving. However, existing approaches such as blood and urine assays are hampered in some testing environments by biohazard risks. A noninvasive, in vivo spectroscopic technique offers a promising alternative, as no body fluids are required. The purpose of this work is to report the results of a 36-subject clinical study designed to characterize tissue alcohol measured using near-infrared spectroscopy relative to venous blood, capillary blood, and breath alcohol. Comparison of blood and breath alcohol concentrations demonstrated significant differences in alcohol concentration [root mean square of 9.0 to $13.5 \mathrm{mg} / \mathrm{dL}$ ] that were attributable to both assay accuracy and precision as well as alcohol pharmacokinetics. A first-order kinetic model was used to estimate the contribution of alcohol pharmacokinetics to the differences in concentration observed between the blood, breath, and tissue assays. All pair-wise combinations of alcohol assays were investigated, and the fraction of the alcohol concentration variance explained by pharmacokinetics ranged from $41.0 \%$ to $83.5 \%$. Accounting for pharmacokinetic concentration differences, the accuracy and precision of the spectroscopic tissue assay were found to be comparable to those of the blood and breath assays. (C) 2009 Society of Photo-Optical Instrumentation Engineers. [DOI: 10.1117/1.3253353]
\end{abstract}

Keywords: alcohol; tissue; near-infrared; pharmacokinetics; blood; reflectance; spectroscopic; noninvasive.

Paper 09267R received Jun. 25, 2009; revised manuscript received Aug. 20, 2009; accepted for publication Aug. 20, 2009; published online Oct. 30, 2009.

\section{Introduction}

\subsection{Alcohol Testing in Society}

As technology and analysis techniques have evolved, the number of biomedical applications of spectroscopy has correspondingly grown. ${ }^{1,2}$ One area that has experienced significant interest and expansion is the noninvasive measurements of analytes in vivo. For example, the noninvasive measurement of glucose has been pursued due to the desire to improve the standard of care for diabetes. However, there are other analytes of similar interest that are amenable to noninvasive spectroscopic measurement. One such analyte is ethyl alcohol.

Although alcohol testing in humans is typically associated with drunk driving, it also plays significant roles in probation monitoring, workplace safety, and emergency medicine. Blood, breath, and urine alcohol measurements are currently performed in these environments to varying degrees. However, these measurements have limitations that manifest differently in each testing environment. For example, in emergency medicine, the concentration of alcohol in a patient is

Address all correspondence to: Trent D. Ridder, TruTouch Technologies, Inc., 800 Bradbury SE, Suite 219, Albuquerque NM, 87106. Tel: 505-272-7050; Fax: 505-272-7083; E-mail: Trent.Ridder@TruTouchTechnologies.com crucial information, as it can influence treatment decisions. ${ }^{3}$ While blood samples can be readily obtained, the time required to receive blood alcohol test results from the hospital or offsite laboratory can delay diagnoses and treatment decisions. Although breath alcohol tests can provide rapid results without sending samples to a laboratory, they are hampered by the need for the patient to breathe into the device, which is a problem for nonresponsive or comatose patients as well as patients without the necessary lung capacity. An alternative means for measuring a patient's alcohol concentration in emergency medicine would be desirable in order to avoid these limitations. A noninvasive, in vivo spectroscopic technique offers promise to achieve this desire, as no bodily fluids are required and results are immediately available, thereby avoiding sample transport and laboratory delays. Furthermore, only passive contact with the patient would be required, which alleviates concerns regarding the responsiveness of the patient.

Another active area in alcohol testing is in home arrest, probation, and work release, where the subject must remain alcohol-free as part of the terms of parole/sentencing. In these situations, an individual is typically tested frequently enough

1083-3668/2009/14(5)/054039/11/\$25.00 @ 2009 SPIE 


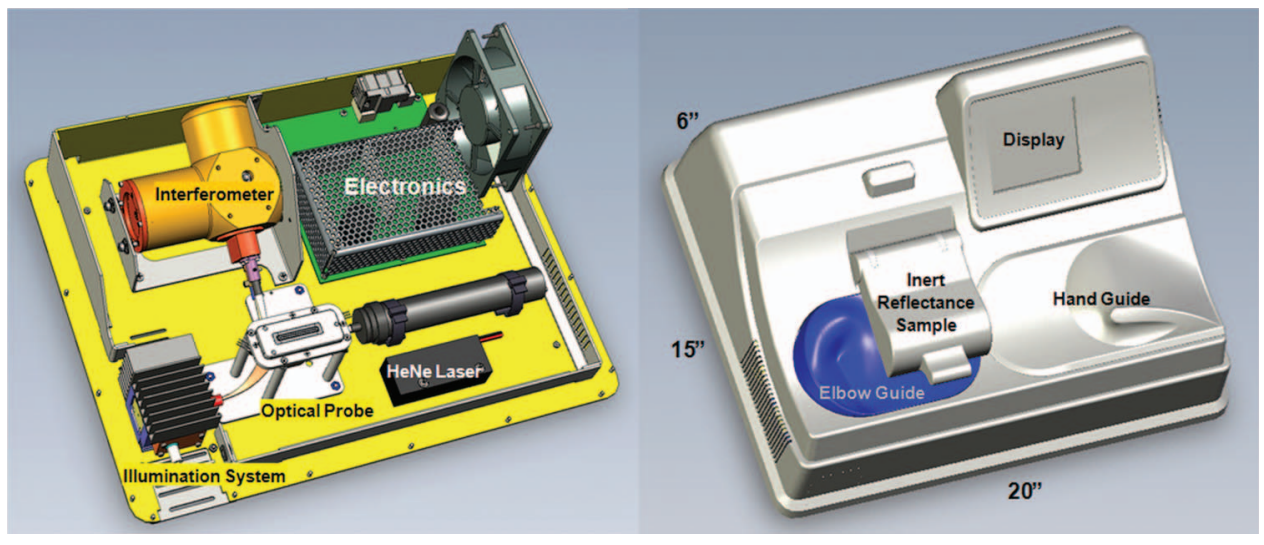

Fig. 1 TTT-1100 system schematic.

that test administration and observation becomes a nontrivial resource constraint. To cater to these environments, existing solutions "enroll" the subject on the instrument with an initial measurement where the blood alcohol concentration is known to be zero, ${ }^{4,5}$ which enables subsequent autonomous measurements when the alcohol test is coupled with biometric identity verification. This approach significantly reduces administrative cost and/or allows for increased testing frequency for greater deterrence. The noninvasive alcohol measurement device discussed in the following is designed to perform in these environments (e.g., the alcohol measurement is combined with identity verification) and is therefore also premised on subject enrollment.

Other alcohol measurement environments may have different motivations for considering a noninvasive measurement, such as concerns over obtaining and handling blood or other bodily fluids. Regardless of their motivations, they all share the same requirement that any new technique, spectroscopic or otherwise, must be characterized relative to the accepted methods currently in practice. This characterization can take many forms, including evaluation of the fundamental theory underlying the measurement as well as clinical evaluation of its performance.

The purpose of this work is to report the results of a clinical study designed to provide characterization of forearm tissue alcohol measured using near-infrared spectroscopy (NIRS) relative to blood and breath alcohol measurements during controlled drinking experiments. Several researchers have used similar clinical studies to investigate the absorption, distribution, metabolism, and elimination (collectively referred to as pharmacokinetics) of alcohol in the body by measuring alcohol concentrations in different compartments of the body (typically via blood and breath assays). ${ }^{6-9}$ These studies have demonstrated that differences in alcohol concentration exist between different bodily fluids, including venous and arterial blood. This work, to our knowledge, provides the first comparison of forearm tissue alcohol to breath, venous, and capillary blood alcohol under similar conditions.

\subsection{NIR Tissue Alcohol Measurement}

The basic physiology of human skin is comprised of epidermal, dermal, and subcutaneous layers, each of which has different properties that influence their relative utility for nonin- vasive alcohol measurements. The epidermis has very little extracellular fluid, and therefore contains minimal information about hydrophilic analytes such as alcohol. The subcutaneous layer is largely comprised of lipids that have low water (and consequently alcohol) solubility, which make it poorly suited to alcohol measurements. However, the dermal layer has high water content ( $\sim 65 \%$ by volume) and an extensive capillary bed conducive to the transport of alcohol, which makes it the important layer of skin tissue for alcohol measurements.

A TTT-1100 (manufactured by TruTouch Technologies, Inc.) was used to measure the tissue alcohol concentration of the volar forearm skin. The TTT-1100 employs NIRS (4000 to $8000 \mathrm{~cm}^{-1}$ ), which is of interest for noninvasive alcohol measurements because it offers specificity for a number of analytes, including alcohol and other organic molecules, while allowing optical path lengths of several millimeters through tissue, thus allowing penetration into the dermal tissue layer, where alcohol is present in the interstitial fluid. ${ }^{10-12}$ A schematic of the TTT-1100 is shown in Fig. 1, and representative forearm tissue spectra are shown in Fig. 2(a).

The TTT-1100 used in this work was calibrated using spectra and blood alcohol reference values obtained from a different subject population on a separate set of instruments. At no time were any of the subjects, nor any data from the device used in this work, incorporated into the calibration regression. The calibration regression was formed using partial least squares (PLS) regression. Additional details regarding the calibration of the TTT-1100 are available in a previous publication. $^{13}$

For illustrative purposes, the 4000 to $8000 \mathrm{~cm}^{-1}$ pure component spectra of ethanol and water [Fig. 2(b)] were used to demonstrate the sensitivity and selectivity of the calibration regression model for alcohol. Information regarding the experimental measurement of the pure components can be found elsewhere. ${ }^{14}$ (Although the pure components of this work spanned the 4000 to $8000 \mathrm{~cm}^{-1}$ region and Ref. 14 used the 4225 to $4625 \mathrm{~cm}^{-1}$ region, the method for determining the pure components was identical.) Because the path lengths encountered in reflectance spectroscopy are not well represented by the fixed path length of a cuvette, an estimate of the effective path length $\left[l_{e f f}\right.$, Eq. (1)] was used to better approximate the wavelength-dependent path lengths encountered in mea- 

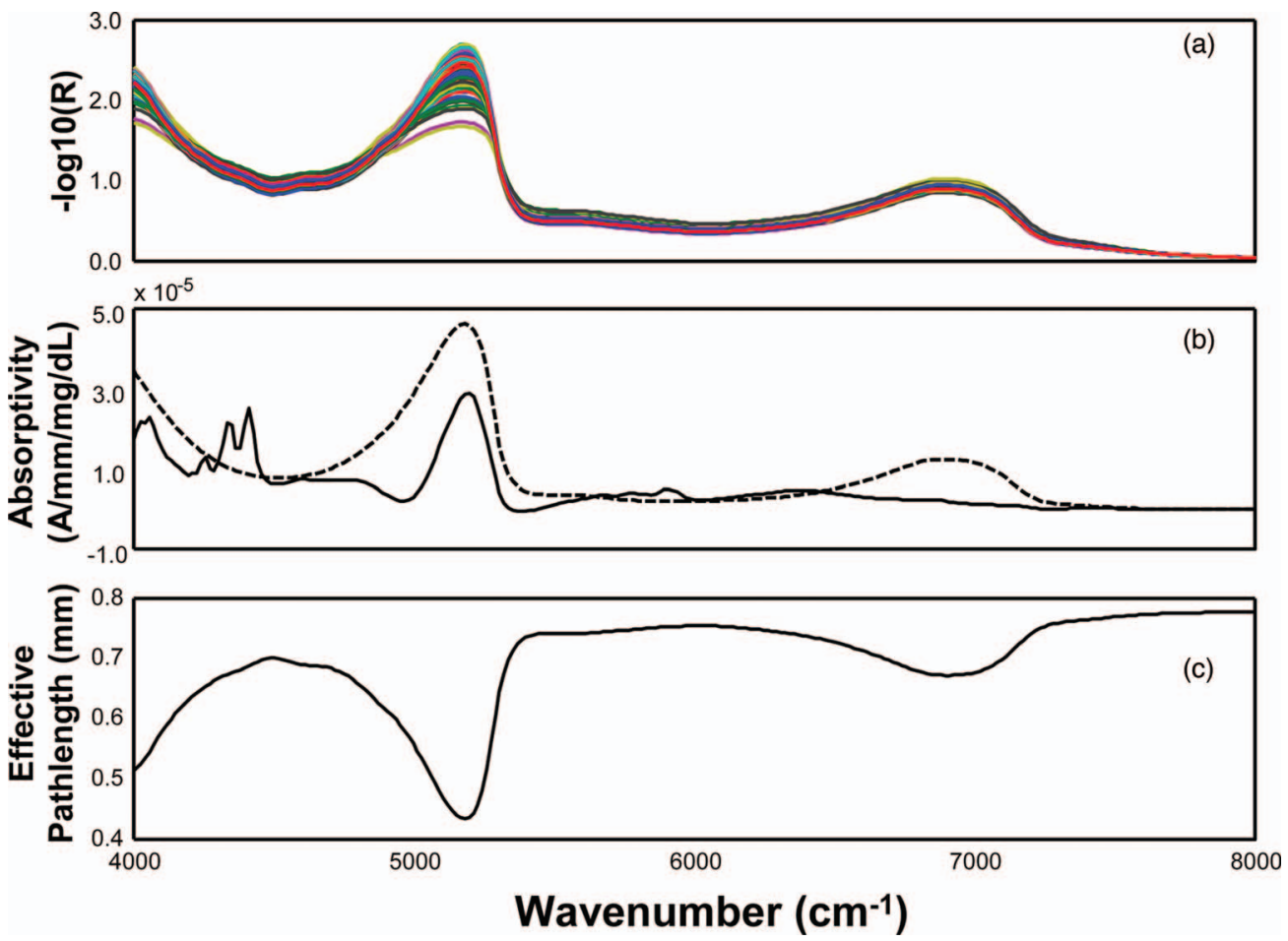

Fig. 2 (a) Log $\log _{10}$-transformed tissue reflectance spectra; (b) pure component spectra of ethanol (-) and water (---); and (c) wavelength-dependent effective path length.

surements of tissue. The effective path length is defined as:

$$
l_{e f f}(\nu)=\frac{\sum_{i=1}^{N} l_{i} e^{-\mu_{a}(\nu) l_{i}}}{\sum_{i=1}^{N} e^{-\mu_{a}(\nu) l_{i}}},
$$

where $\mu_{a}$ is the absorption coefficient at wave number $\nu, l_{i}$ is the path length through tissue of photon $i$ as determined by Monte Carlo simulation, ${ }^{14}$ and $N$ is the total number of collected photons. The effective path length, $l_{\text {eff }}$, depends on the scattering properties of skin, the geometry of the tissue optical probe (both of which are incorporated into the determination of $l$ in the Monte Carlo simulation), and the absorptivities of major absorbers, $\mu_{a}$, which are dominated by water in tissue measurements. Figure 2(c) shows an estimate of the TTT1100 effective path length.

Figure 3 shows the projections of the effective path length-corrected ethanol (solid) and water (dashed) pure components onto the calibration regression coefficients versus the number of PLS factors in the calibration model. The ethanol projection is indicative of the calibration sensitivity and approaches $1 \mathrm{mg} / \mathrm{dL}$ at approximately 25 factors. This result is a strong indication that the TTT-1100 ethanol calibration model, developed from noninvasive spectroscopic data and associated ethanol reference values, is indeed calibrated to ethanol absorption features.

Furthermore, the projection of the water pure component stays very near zero throughout all factors examined. This result is not surprising, as water is a strong absorber that is present at high concentration in tissue. Thus, the calibration regression must remain highly selective against it in order to minimize the mean squared error. ${ }^{13}$ The TTT-1100 model does not, therefore, exploit water displacement effects as a surro- gate for tissue ethanol changes. Additional discussion and investigations of the NIR instrumentation, calibration method, and initial characterization of the method's sensitivity and selectivity for alcohol can be found elsewhere. ${ }^{13-15}$

At this point, it is worth briefly discussing the properties of the noninvasive tissue alcohol measurement in this work relative to another recently developed technology. On first glance, one might assume that "tissue" and "dermal" alcohol equate to "transdermal" alcohol. However, transdermal alcohol measurements such as those described by Swift ${ }^{16,17}$ refer to alcohol vapor that has evaporated from perspiration excreted at

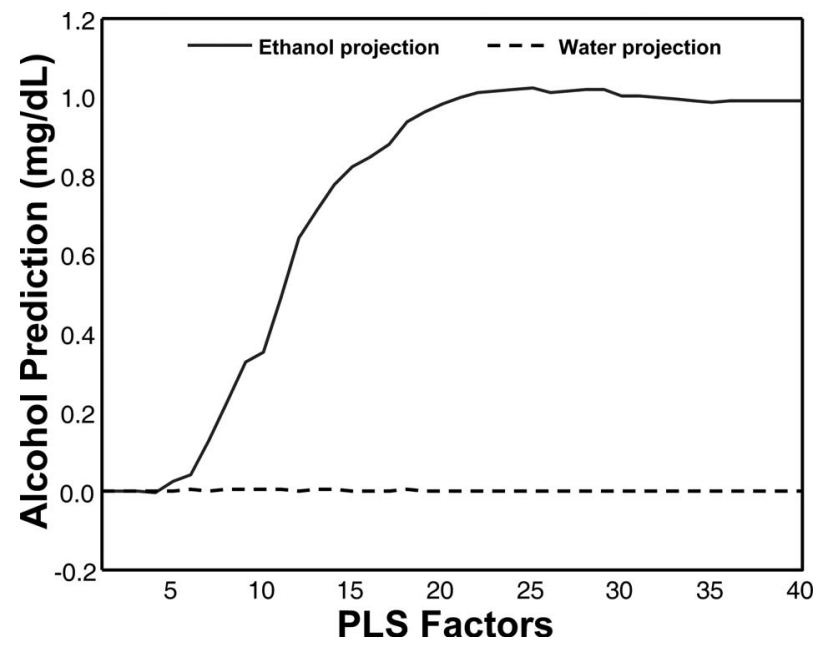

Fig. 3 Effective path length corrected ethanol and water pure component projections onto calibration regression coefficients. 
Ridder, Ver Steeg, and Laaksonen: Comparison of spectroscopically measured tissue alcohol concentration to blood...

Table 1 Demographics of clinical study participants.

\begin{tabular}{|c|c|c|c|c|c|}
\hline Ethnicity & Caucasian & Hispanic & $\begin{array}{l}\text { African } \\
\text { American }\end{array}$ & $\begin{array}{c}\text { Native } \\
\text { American }\end{array}$ & \\
\hline \# subjects & 22 & 10 & 1 & 3 & \\
\hline Age (years) & $21-30$ & $31-40$ & $41-50$ & $51-60$ & $>60$ \\
\hline \# subjects & 20 & 6 & 4 & 3 & 3 \\
\hline $\mathrm{BMI}\left(\mathrm{kg} / \mathrm{m}^{2}\right)$ & $16-20$ & $21-25$ & $26-30$ & $31-35$ & $>36$ \\
\hline \# subjects & 7 & 11 & 12 & 3 & 3 \\
\hline Gender & Male & Female & & & \\
\hline \# subjects & 18 & 18 & & & \\
\hline
\end{tabular}

the tissue surface, and not the alcohol concentration in situ within the dermis. In contrast, the tissue measurement discussed in this work uses an optical probe to direct NIR radiation into the dermal layer of the tissue and collect the radiation that is diffusely reflected back to the tissue surface, thereby directly interrogating the aqueous alcohol present in the interstitial fluid of the demis. Hence, there is a critical distinction between the previously mentioned transdermal methods and the tissue alcohol measurement described in this work.

\section{Methods}

\subsection{Clinical Study Description}

Alcohol excursions were induced in 36 subjects (demographics shown in Table 1) at the University of New Mexico Clinical Trial Center following overnight fasts in order to compare tissue alcohol concentration to blood and breath alcohol concentrations. Written consent was obtained from each participant following full explanation of the IRB-approved protocols (University of New Mexico School of Medicine Human Research Review Committee). Baseline capillary blood, venous blood, breath, and noninvasive tissue alcohol measurements (details described in the following) were taken upon arrival in order to verify zero initial alcohol concentration in all subjects.

The alcohol dose for each subject was ingested orally with a target peak blood alcohol concentration of $100 \mathrm{mg} / \mathrm{dL}$ $(0.10 \%)$ for all subjects. The mass of the alcohol dose was calculated for each subject using an estimate of total body water based on gender and body mass. ${ }^{18}$ An alcohol dose limit of $110 \mathrm{~g}$ was imposed to prevent overdosing obese subjects whose weight tended to overestimate their total body water. The alcohol doses were mixed with orange juice $(50 \%$ by volume) to ease consumption.

Upon complete ingestion of the alcohol dose, repeated cycles of breath, capillary blood, venous blood, and tissue alcohol measurements were acquired $(\sim 20$ minutes per cycle) from each subject until his or her blood alcohol concentration reached its peak and then declined below $20 \mathrm{mg} / \mathrm{dL}(0.02 \%)$. Under these conditions, the average excursion lasted approximately $5 \mathrm{~h}$ and yielded approximately
11 sets (minimum of 8 and maximum of 18) of tissue, blood, and breath alcohol measurements per subject. A total of 398 sets of measurements were acquired from the 36 subjects.

\subsection{Blood Sample Acquisition and Alcohol Assays}

A catheter was inserted into the right median cubital vein of each subject and was used to collect venous blood samples. The venous blood samples were drawn into gray top vacuettes containing sodium fluoride to inhibit glycolysis and potassium oxalate to prevent coagulation. Capillary blood samples were obtained from the fingertip using lancing devices and microcapillary tubes. Alcohol assays were performed on the collected venous blood samples using two techniques: an alcohol oxidase-based method performed on site (described in the following), and headspace gas chromatography (GC) analysis performed at TriCore Reference Laboratories (Albuquerque, New Mexico). Due to their smaller blood volume, the capillary blood samples were analyzed using only the on-site enzymatic method.

The TriCore GC assay used a Hewlett Packard 5890 II gas chromatograph with a flame ionization detector (FID). The carrier gas was helium, and the separation column was a J\&W DB ALC2. The instrument was calibrated using a commercially purchased $50 \mathrm{mg} / \mathrm{dL}$ ethanol calibration standard (Cerilliant part A-057) and verified using a gravimetrically prepared calibration test sample containing $100 \mathrm{mg} / \mathrm{dL}$ ethanol. Expired whole blood (verified alcohol concentration of $0 \mathrm{mg} / \mathrm{dL}$ ) was used as a control. The sample volume was $100 \mu \mathrm{L}$ for the calibration standard, calibration test sample, control, and collected venous blood samples, and $100.0 \mu \mathrm{L}$ of tert-butanol was added as an internal standard to all samples.

The alcohol oxidase assays were performed using a Yellow Springs Incorporated (YSI) 2700 Select Biochemistry Analyzer present within the clinical laboratory. The YSI 2700 was calibrated using certified standards from a calibration kit (YSI part number 2790). The calibration was verified every $15 \mathrm{~min}$ throughout the study using a certified calibration test sample (included in YSI Part Number 2790). YSI alcohol assays were performed on all capillary and venous blood samples immediately after their acquisition (no more than $5 \mathrm{~min}$ from acquisition to assay). 


\subsection{Breath Sample Acquisition and Alcohol Assays}

Breath alcohol concentrations were used in this work as an alternative means for investigating the arterial blood space (acquisition of arterial blood carries greater risk to the subject due to the greater arterial blood pressure). Several studies have shown that breath alcohol closely tracks the arterial blood space. ${ }^{19-21}$

A factory-calibrated Drager Alcotest 6510 was used to acquire all breath alcohol measurements. A new mouthpiece was used for each subject (part A6510). As breath testers measure the alcohol concentration present in the breath, a multiplicative conversion factor (referred to as the blood-breath ratio, or BBR) must be applied that relates the much lower breath alcohol concentration to blood alcohol concentration. Although the blood-breath ratio is known to vary between people (1981 to 2833), ${ }^{22}$ in the United States it is mandated to be 2100 for evidentiary breath alcohol measurements. However, studies have shown that a value of 2300 better represents the average BBR across a broad subject population. ${ }^{22,23}$ As the objective of this work is to compare tissue alcohol concentration to the concentrations in blood, the recorded breath alcohol values (with implicit $2100 \mathrm{BBR}$ ) were converted to concentrations corresponding to a BBR of 2300 in order to better represent the arterial blood space.

\subsection{Spectroscopic Tissue Alcohol Measurement}

The tissue alcohol measurements were acquired using a 3-min measurement time. The only requirement of the spectroscopic measurement was passive contact between the volar forearm of the subject and the optical probe of the device during the 3-min period. The stability of the spectroscopic device was verified on 30-min intervals throughout the study using a spectroscopically and environmentally inert reflectance sample placed in contact with the optical probe between subject measurements.

For the purposes of the clinical study in this work, the baseline NIR measurements acquired prior to the alcohol excursions were used to "enroll" their associated subjects. Enrollment was achieved by subtracting each subject's baseline spectrum (with known zero alcohol concentration) from the remaining tissue spectra acquired during his/her alcohol excursion experiment. The subtraction of the enrollment spectrum removes major spectral attributes that are unique to each subject while retaining any analyte variation within the subjects that occurs over the course of the experiment (e.g., changes in ethanol concentration). The calibration data were comprised of spectroscopic measurements from subjects that were enrolled in the same manner. Similar methods for in vivo spectroscopy have been previously described. ${ }^{24}$ The experimental data were imported into MATLAB 7.5, which was used to perform all analyses and generate the results presented in subsequent sections.

\section{Results and Discussion}

\subsection{Comparison of Reference Alcohol Concentrations}

A challenge commonly encountered in the evaluation of a new measurement technique is the selection of a reference method for comparison. In the case of the NIR measurement of tissue alcohol discussed in the present work, the selection of a reference is further complicated by the fact that there are no alternative techniques for measuring dermal alcohol. As a result, regardless of what reference method is chosen for comparison, the observed differences will be comprised of two significant sources: (1) the accuracy and precision of the NIR and reference assay methods and (2) true physiologic differences in alcohol concentration between the compartments/ fluids of the body.

Not only are these two differences present when comparing dermal alcohol concentration to a selected reference, they are also present when comparing two alcohol reference assays to each other. Several researchers have demonstrated these differences by comparing contemporaneous venous blood, breath, and arterial blood alcohol concentrations during similar dosing studies. . $^{9,19,21,23}$

As capillary blood, venous blood, and breath alcohol (arterial blood surrogate) were collected in the clinical study, this allowed examination of the differences observed between reference measurements for a fixed set of study conditions. Furthermore, the venous blood samples were assayed by both headspace GC and enzymatic techniques, which offers the ability to investigate the precision and accuracy of the assay methods independent of pharmacokineticss.

Consequently, comparison of the reference measurements to each other can serve as a starting point for the evaluation of the spectroscopic alcohol measurement by providing insights into relative accuracy and precision of the assay methods as well as the pharmacokinetic differences observed between the reference assays. Figure 4 depicts several comparisons of the reference alcohol measurements acquired during the clinical study that are discussed in the following.

\subsubsection{Venous blood alcohol: assay method comparison}

Figure 4(a) depicts the venous alcohol concentrations obtained from the enzymatic assay versus the venous alcohol concentrations obtained by headspace GC analysis. Ideally, all points in Fig. 4(a) would fall on the dotted line as the samples were identical in both cases. The fact that the two reference techniques do not perfectly agree indicates the presence of error in the concentration measurements. Visual inspection indicates that there is a systematic difference between the measurement methods, as the enzyme concentrations exhibit an $8.9 \mathrm{mg} / \mathrm{dL}$ positive bias relative to the GC concentrations. From these data alone, it is not possible to definitively determine the origins of the bias (e.g., both methods may have a finite bias, but plotted against each other, the difference in biases is $8.9 \mathrm{mg} / \mathrm{dL}$ ). Furthermore, acknowledging the presence of the bias, there remains a distinct scatter between the methods, as evidenced by a bias-corrected root mean square (RMS) difference between methods of $8.4 \mathrm{mg} / \mathrm{dL}$. Given that both the enzymatic and the GC measurements were performed on calibrated instruments by trained clinical and laboratory staff, the presence of significant systematic and precision differences is an illuminating result that provides background for how well assay methods agree under experimental conditions. 


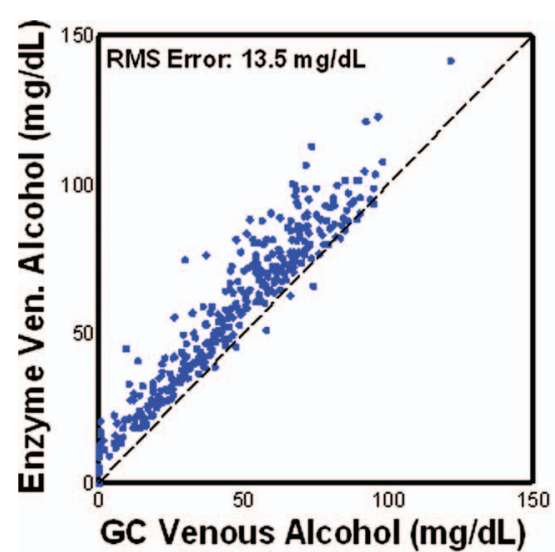

(a)

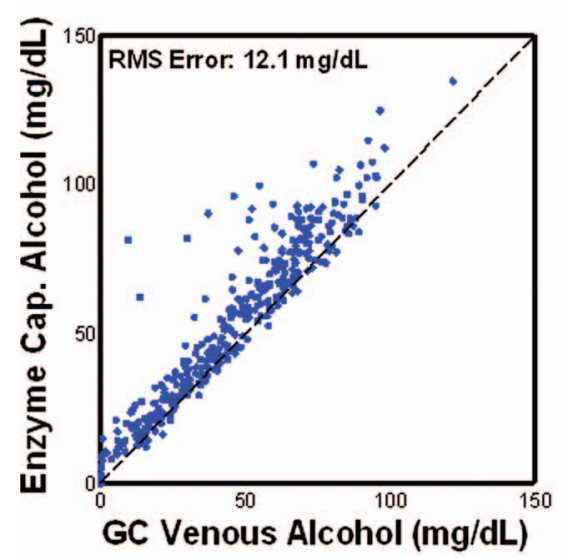

(d)

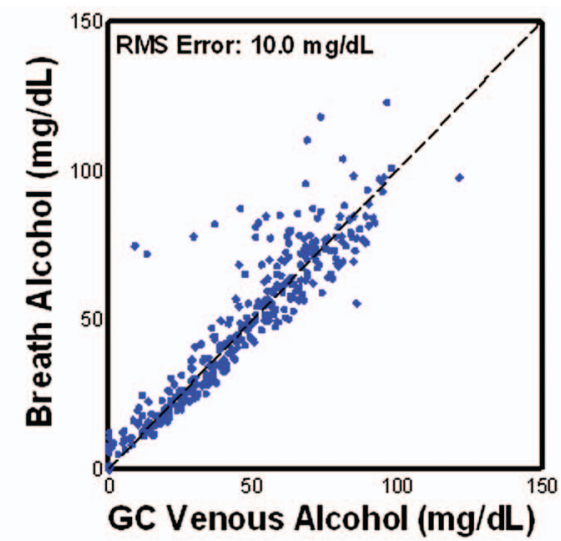

(b)

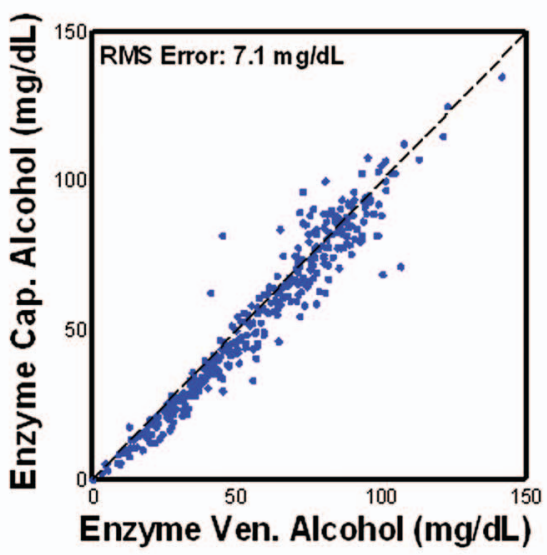

(e)

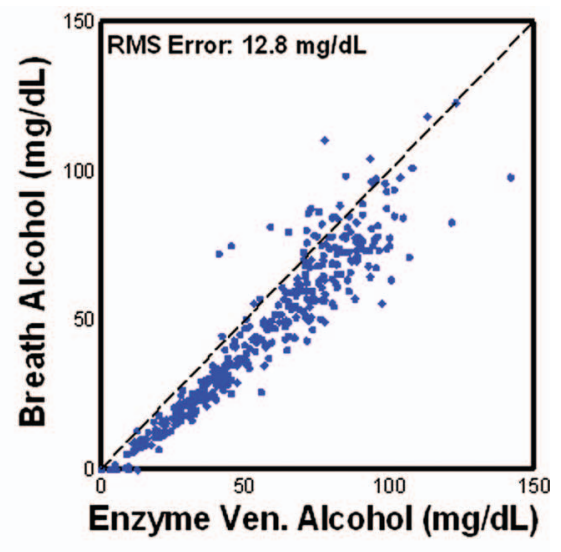

(c)

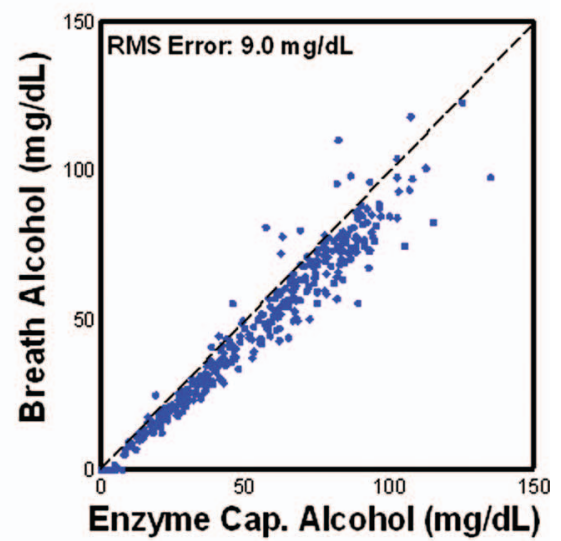

(f)

Fig. 4 Comparison of alcohol concentrations from reference methods.

\subsubsection{Comparison of venous blood, capillary blood, and breath alcohol concentrations}

The previous examination of the venous blood samples demonstrated that the alcohol concentrations obtained from the same samples by two different techniques contain both systematic and precision differences. Certainly, similar sources of measurement differences will be present in the capillary blood and breath samples. However, when comparing alcohol concentrations across sample types (e.g., venous to capillary or venous to breath), the influence of alcohol pharmacokinetics must also be considered.

Figures 4(b) and 4(c) show a comparison of breath alcohol and contemporaneously acquired venous blood alcohol measured via headspace GC [Fig. 4(b)] and the enzyme assay [Fig. 4(c)]. There are several measurements where the difference between breath and venous blood is large. Examination of Figs. 4(b) and 4(c) shows that the points that exhibit the largest differences between methods generally have a breath concentration that is larger than the venous blood concentration. For example, there are points in Fig. 4(b) that exhibit breath alcohol concentrations of approximately $75 \mathrm{mg} / \mathrm{dL}$, while the corresponding venous alcohol concentrations are below $25 \mathrm{mg} / \mathrm{dL}$. As breath alcohol is related to arterial alcohol, these differences are indicative of concentration gradients between the arterial and venous blood spaces. This effect can be more easily observed by examining the alcohol concentrations over time acquired from a few exemplary subjects (Fig. 5).

Examination of Fig. 5 demonstrates that upon alcohol consumption, the breath (arterial) alcohol concentration is the first to rise, with the venous alcohol generally rising later and at a slower rate. This arterial-venous phenomenon has been observed in several clinical studies ${ }^{19,25}$ that have shown that a significant portion of the disagreement between breath and venous alcohol concentrations is attributable to pharmacokinetics (e.g., true differences in alcohol concentration) rather than the accuracy or precision of the assay methods themselves.

Figures 4(d)-4(f) show the remaining pair-wise comparisons of the reference alcohol assays. Examination of the relationship between capillary blood and venous blood [Figs. 4(d) and $4(\mathrm{e})]$ indicates that its behavior is similar to that observed between breath and venous alcohol concentration [Figs. 4(b) and $4(\mathrm{c})$ ] in that several data points exhibit large concentration differences, with capillary blood concentration being greater than venous concentration. Furthermore, Fig. 4(e) exhibits a smaller RMS error (7.1 mg/dL) than Fig. 4(d) $(12.1 \mathrm{mg} / \mathrm{dL})$ due to the enzyme assay method being common to both blood sample types. It should be noted that systematic errors from the enzyme assay method do contribute to 

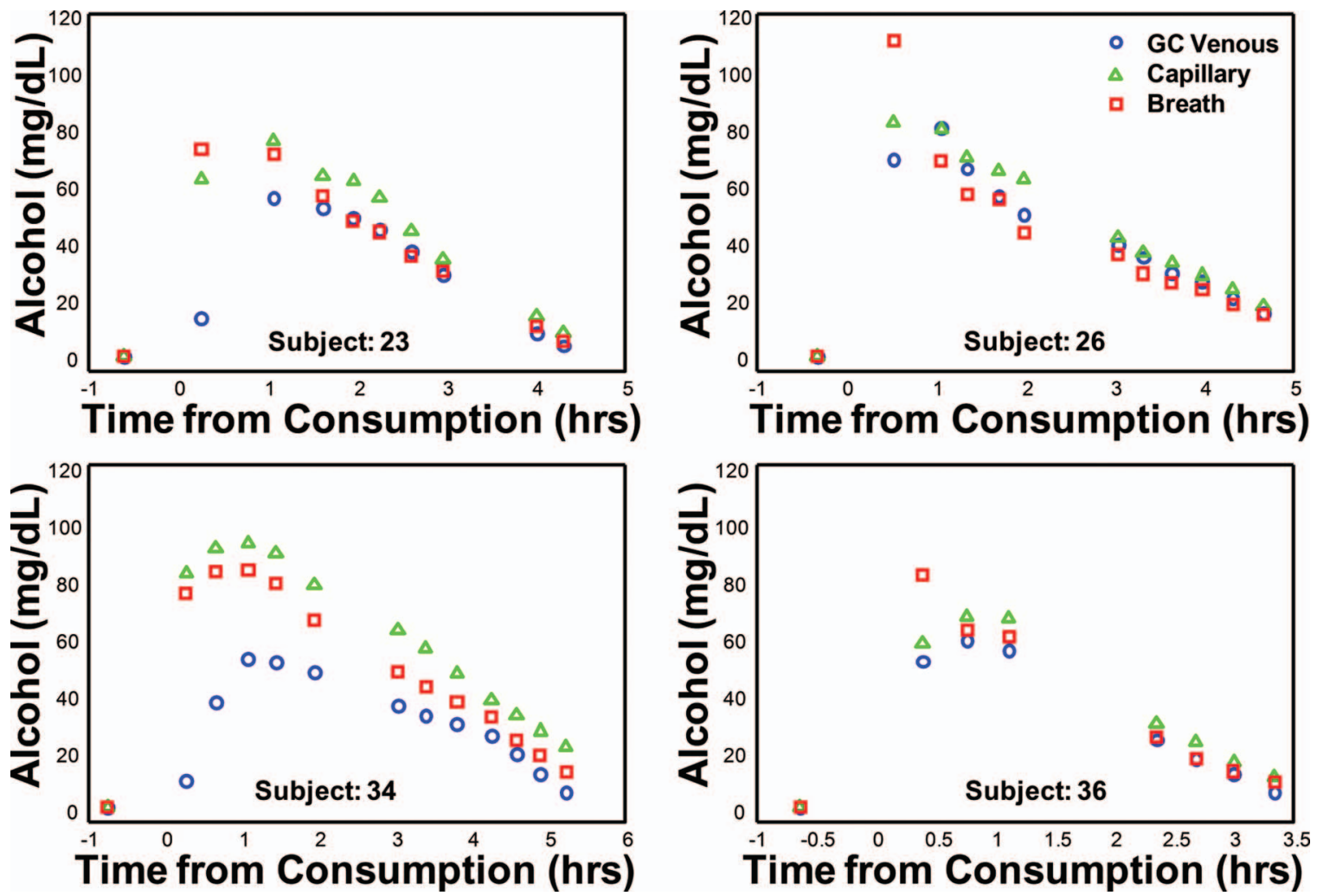

Fig. 5 Reference alcohol concentrations over time for selected subjects.

the absolute location of each point in Fig. 4(e). However, as the systematic error is common to both sample types, its contribution lies along the diagonal, which reduces its impact on the RMS concentration difference. This suggests that $7.1 \mathrm{mg} / \mathrm{dL}$ is a reasonable estimate of the assay-independent RMS concentration difference between venous and capillary blood. Figure 4(f) shows that significant differences between capillary blood and breath alcohol are also apparent, but generally smaller in magnitude than the comparisons of other sample types. This suggests that the finger capillary blood is more similar to the arterial blood space than the venous blood space in a pharmacokinetic sense.

\subsection{Quantification of Pharmacokinetic Differences}

The comparison of the alcohol reference assays indicates that the different compartments exhibit different concentrations over time. Furthermore, tissue (skin or otherwise) represents yet another compartment, which suggests that pharmacokinetic differences will also manifest when comparing tissue alcohol measurements to those obtained from other compartments or bodily fluids. ${ }^{26}$ A means for quantifying pharmacokinetic differences is desirable in order to isolate concentration differences arising from the assay techniques from those that are caused by pharmacokinetics.

The concentration differences between blood types within study participants have been examined using compartmental analysis that uses a first-order kinetic model [Eq. (2)] to explain the equilibration of alcohol between compartments: ${ }^{6-9}$

$$
\frac{\mathrm{d} C_{2}}{\mathrm{~d} t}=k_{12}\left(C_{1}-C_{2}\right)
$$

where $C_{1}$ is the alcohol concentration in compartment 1 at time $t, C_{2}$ is the concentration in compartment 2 at time $t, k_{12}$ is the first-order rate constant in $\mathrm{min}^{-1}$ that regulates the transfer of alcohol between compartments, and $\left(\mathrm{d} C_{2} / \mathrm{d} t\right)$ is the rate of change of alcohol concentration in compartment 2 in $\mathrm{mg} / \mathrm{dL} / \mathrm{min}$. In a general sense, larger values of $k_{12}$ indicate faster equilibration between compartments, with $\infty$ representing instant equilibration and 0 representing no equilibration.

Pharmacokinetic rate constants were estimated for each pair of compartments for each subject using the experimentally measured alcohol concentrations and Eq. (2). Nonlinear least squares regression was used to estimate the rate constants by numerical integration of Eq. (2) (via MATLAB). The nonlinear regression can be considered as an iterative process. First, Eq. (2) was integrated using an arbitrarily chosen initial value for the rate constant $\left(k_{12}\right)$ and the experimentally measured compartment 1 concentrations $\left(C_{1}\right)$ in order to obtain estimates of compartment 2 concentration $\left(\hat{C}_{2}\right)$. Second, the mean squared error of $\hat{C}_{2}$ relative to the experimental compartment 2 concentrations $\left(C_{2}\right)$ was used to select a new rate constant value for the next iteration. Thus, the objective of the nonlinear regression was to determine the value of $k_{12}$ that minimized the mean squared error between the estimated compartment 2 concentrations $\left(\hat{C}_{2}\right)$ and the experimentally measured concentrations $\left(C_{2}\right){ }^{8}$ For each pair of compart- 

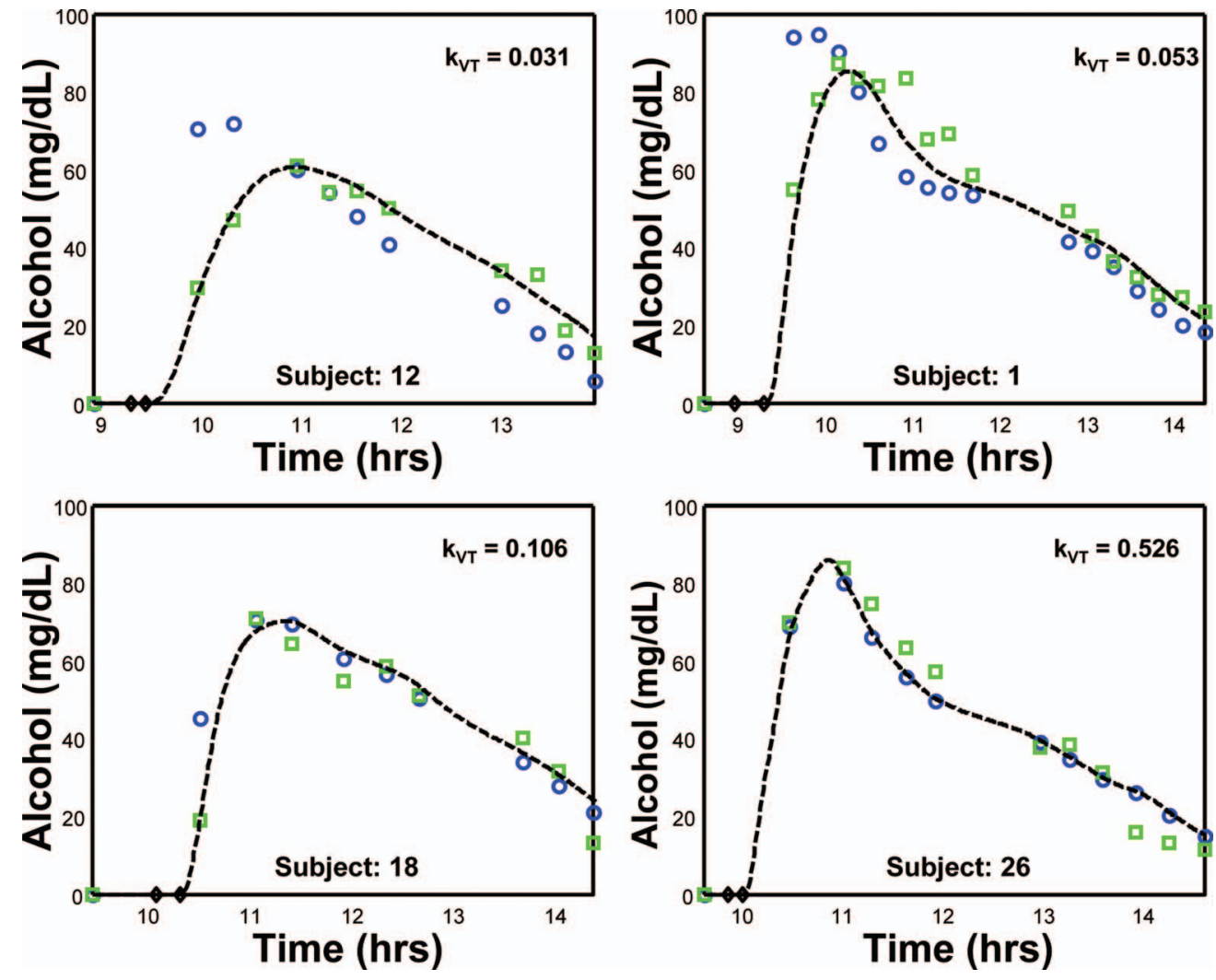

Fig. 6 GC venous alcohol $(\bigcirc)$, tissue alcohol $(\square)$, and kinetic parameter fits (---) for exemplary subjects.

ments, the subscript 12 was replaced by letters denoting the corresponding compartments with $A, C, V$, and $T$ denoting arterial (breath), capillary, venous, and tissue, respectively. For example, $k_{A C}$ is the rate constant, where $C_{1}$ is the arterial compartment and $C_{2}$ is the capillary compartment.

Figure 6 shows exemplary $k_{V T}$ estimates from four subjects, where $C_{1}$ was $\mathrm{GC}$ venous blood alcohol and $C_{2}$ was the noninvasive tissue alcohol measurement. The influence of the magnitude of $k_{V T}$ on the venous-tissue mismatch is evident across the four subjects as the difference between the blood and tissue alcohol becomes exponentially more pronounced as
$k_{V T}$ linearly decreases. The results in Fig. 6 provide confidence that the first-order model in Eq. (2) is appropriate, as it yields reasonable estimates of the tissue alcohol concentrations for each of the subjects investigated.

Table 2 summarizes the rate constant estimates obtained in this work. Each entry in Table 2 was generated from 36 rate constant estimates ( 1 from each of the 36 study participants). Furthermore, the underlying distribution of each set of estimates was determined to be lognormal at $95 \%$ confidence. Consequently, the parameterization in Table 2 reflects the lognormal distribution of the rate constant estimates. The mean

Table 2 Summary of pharmacokinetic fits.

\begin{tabular}{lcccccc}
\hline & $\begin{array}{c}\text { Most } \\
\text { probable } \\
\text { value }\end{array}$ & $\begin{array}{c}\text { lognormal } \\
\mu\end{array}$ & $\begin{array}{c}\text { lognormal } \\
\sigma\end{array}$ & Mean & Variance & $\begin{array}{c}\% \text { Variance } \\
\text { explained } \\
\text { [Eq. (3)] }\end{array}$ \\
\hline$k_{12}$ & 0.271 & -0.15 & 1.08 & 1.54 & 5.26 & 41.0 \\
$k_{A V}$ & 0.097 & -1.39 & 0.98 & 0.40 & 0.26 & 68.2 \\
$k_{A T}$ & 0.032 & -3.32 & 0.36 & 0.039 & 0.0002 & 78.3 \\
$k_{C V}$ & 0.106 & -0.89 & 1.17 & 0.81 & 1.94 & 64.2 \\
$k_{C T}$ & 0.031 & -3.29 & 0.44 & 0.041 & 0.0004 & 83.5 \\
$k_{V T}$ & 0.035 & -2.98 & 0.62 & 0.062 & 0.002 & 75.3 \\
\hline
\end{tabular}


and variance of the distribution can be determined from $\mu$ and $\sigma$ by $e^{\mu+\sigma^{2} / 2}$ and $\left(e^{\sigma^{2}}-1\right) e^{2 \mu+\sigma^{2}}$, respectively.

Table 2 also shows the percent variance explained by the first-order kinetic model. These were calculated using Eq. (3) and indicate the percentage of the concentration variance between the experimentally measured alcohol concentrations that is attributable to the pharmacokinetic differences between the compartments:

$$
\% \sigma_{\text {explained }}^{2}=100\left[\frac{\sum\left(\hat{C}_{2}-C_{1}\right)^{2}}{\sum\left(C_{2}-C_{1}\right)^{2} / n}\right],
$$

where $n$ is the number of measurements; $C_{1}$ and $C_{2}$ are the experimentally measured alcohol concentrations from compartments 1 and 2, respectively; and $\hat{C}_{2}$ are the fit concentrations of compartment 2 at the times of $C_{2}$ that were obtained using the estimated rate constant $\left(k_{12}\right), C_{1}$, and numerical integration of Eq. (2).

Examination of Table 2 shows that a significant fraction of the concentration variance of all compartment combinations is attributable to alcohol pharmacokinetics. In particular, comparison of tissue alcohol to any of the other compartments shows that at least $75 \%$ of the concentration variance is explained by the first-order model. It is also interesting to note that the experimentally determined values of $k_{V T}$ determined in this work (most probable value of $0.035 \mathrm{~min}^{-1}$ and a mean of $0.062 \mathrm{~min}^{-1}$ ) agree very well with those estimated by Norberg et al., ${ }^{9}$ who had no direct assay for the tissue compartment (mean $k_{V T}$ of $0.038 \mathrm{~min}^{-1}$ ). (Norberg's Eq. (3): $V_{T}\left(d C_{T} / d t\right)=C L_{d} C_{V}-C L_{d} C_{T}$. Upon rearrangement, Norberg's $C L_{d} / V_{T}$ is equal to $k_{V T}$ of this work. The mean $k_{V T}$ of $0.038 \mathrm{~min}^{-1}$ from Norberg was obtained using the values in Table $1 \mathrm{~b}$ by unit conversion of the $C L_{d}$ to liters and division by $\left.V_{T}\right)$.

\subsection{Comparison of Tissue Alcohol to Reference Methods}

An important question is "How well does the noninvasive NIR technique measure tissue alcohol concentration?" Because there is no other method for determining dermal alcohol concentration, another reference such as venous alcohol concentration must be used with the acknowledgement that true differences in alcohol concentration will be present between them. As a result, the concentration differences observed will be a combination of the errors of the two measurement devices and pharmacokinetic effects.

Equation (2) shows that the concentration differences between compartments are largest when the rate of change is greatest. In the data collected in this work, the intent of the study design was to rapidly bring the participants to $100 \mathrm{mg} / \mathrm{dL}$ in order to maximize the rate of change and improve the estimates of the rate constants. (See Fig. 6, which shows the venous and NIR tissue alcohol concentrations obtained from four study participants.) However, once each subject reached a peak alcohol concentration and began to decline, the rates of change were significantly lower (but nonzero) due to the zero-order elimination of alcohol by the liver. This suggests that the latter portion of each subject's excursion data has less pharmacokinetic error and can help to better isolate the accuracy and precision of the assay methods from pharmacokinetic differences.

In order to achieve this, each subject's alcohol excursion was divided into two periods: absorption and elimination. For the purposes of this work, the absorption period was deemed to have concluded at the point in time following the peak venous alcohol concentration, where tissue alcohol was equal to venous alcohol concentration. However, it is important to note that pharmacokinetic differences between tissue and reference alcohol concentrations still exist, as the rates of changes in all compartments are not zero during the elimination period. In other words, some pharmacokinetic error is an unavoidable consequence of not having an alternative dermal alcohol assay. Figure 7 shows the NIR tissue alcohol concentrations versus GC venous alcohol concentration measured during the 36 subjects' elimination periods $(n=267)$ along with the corresponding $R M S$ difference in concentration.

As mentioned earlier, the elimination phase RMS concentration differences in Fig. 7 (also shown in Table 3 under $\left.R M S_{E x p}\right)$ are comprised of assay error and pharmacokinetic differences. In order to investigate the contributions of those two sources to $R M S_{\text {Exp }}$, estimated tissue concentrations $\left(\hat{C}_{T}\right)$ were determined for each subject by integrating Eq. (2) using the reference alcohol concentrations and previously estimated rate constants. For example, the measured venous blood concentrations and associated $k_{V T}$ for a given subject were used to obtain corresponding tissue concentration estimates $\left(\hat{C}_{T}\right)$. The $R M S$ difference between the estimated elimination phase tissue concentrations and reference values was then calculated for each tissue/reference combination $\left(R M S_{\text {Fit }}\right.$ in Table 3). With the assumption that the pharmacokinetic error is equal in both $R M S_{E x p}$ and $R M S_{F i t}$, estimates of the combined assay error $\left(R M S_{\text {Assay }}\right)$ were obtained by variance subtraction of $R M S_{\text {Fit }}$ from $R M S_{\text {Exp }}$ (rightmost column of Table 3). Estimates of $R M S_{\text {Assay }}$ for the additional pairwise combinations of alcohol references were also determined using the same approach (breath versus capillary- $6.6 \mathrm{mg} / \mathrm{dL}$; breath versus GC venous $-5.4 \mathrm{mg} / \mathrm{dL}$; and capillary versus GC venous $-5.1 \mathrm{mg} / \mathrm{dL}$ ). The $R M S_{\text {Assay }}$ values observed for the combinations of reference methods fell within a range of 5.1 to $6.6 \mathrm{mg} / \mathrm{dL}$, while those involving tissue had a range of 4.8 to $8.0 \mathrm{mg} / \mathrm{dL}$. The observed $R M S_{\text {Assay }}$ ranges may suggest similar assay error contributions for the tissue and reference measurement methods.

\section{Conclusion and Future Directions}

The first-order diffusion model employed in this work was shown to reasonably explain intercompartment alcohol concentrations observed during controlled drinking studies, which is consistent with prior application of first-order models in alcohol pharmacokinetic research. Furthermore, in this work, the first-order model was applied to experimentally measured tissue alcohol concentrations, which showed that, while the diffusion constants were smaller (larger kinetic difference), the first-order model remained a valid means for explaining the concentration differences between tissue blood alcohol concentrations. Accounting for pharmacokinetic concentration differences, the accuracy and precision of the spec- 

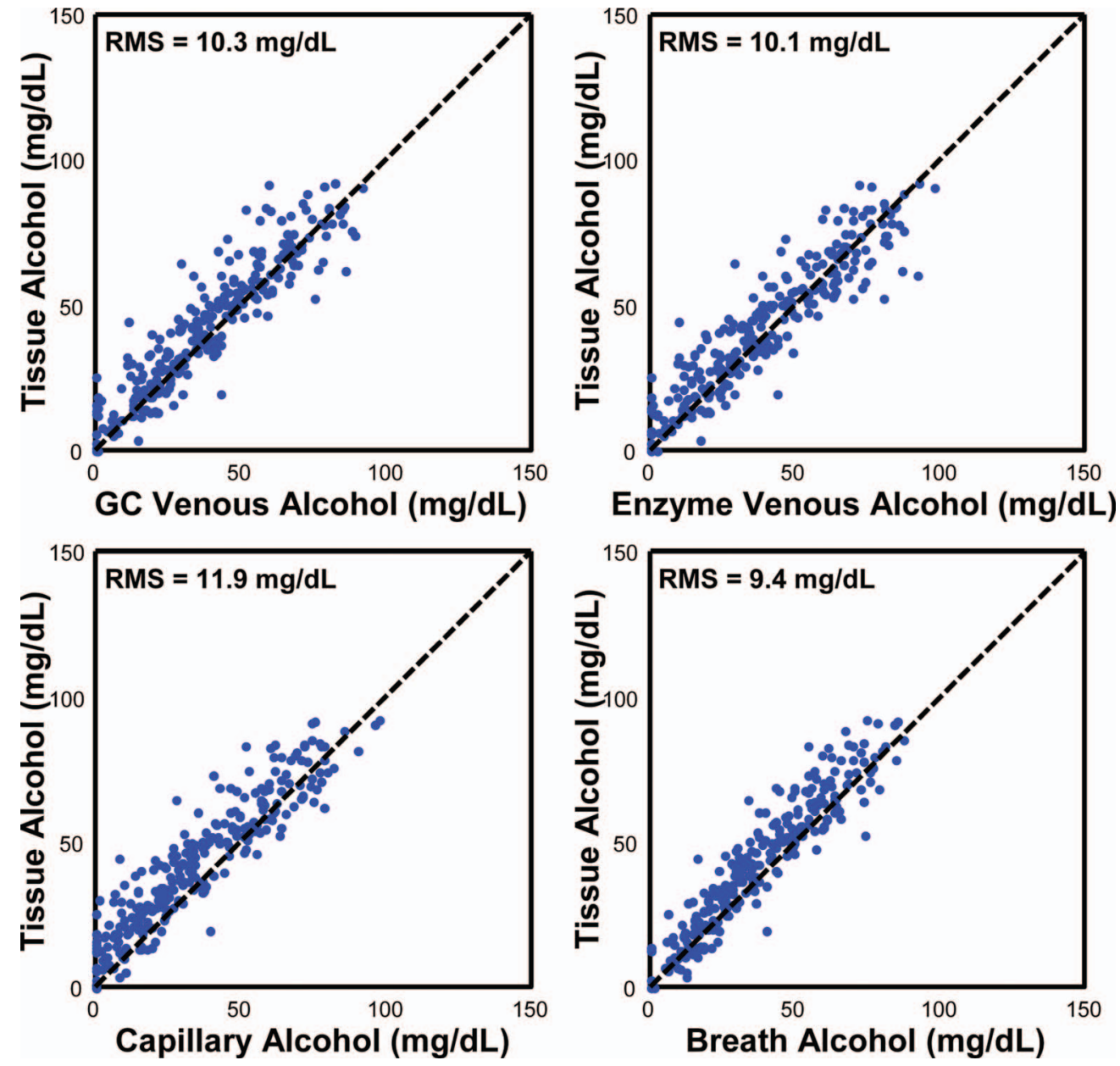

Fig. 7 Post-equilibrium tissue alcohol versus reference alcohol assays.

troscopic tissue assay was found to be comparable to those of the blood and breath assays.

This work yielded several directions for future efforts including demographic and environmental analysis of the tissue pharmacokinetic parameters, comparison of pharmacokinetic parameters at multiple tissue locations, and the potential to apply the first-order model as a means for obtaining improved reference values for formation of the tissue alcohol calibration model.

Demographics analysis of pharmacokinetic parameters is of interest, as it can provide insight into the role of age, gender, and ethnicity on alcohol distribution in the body. Further- more, environmental variables such as temperature and humidity are also important, particularly for the tissue compartment, as they can alter blood perfusion and potentially influence the pharmacokinetic parameters.

The tissue alcohol concentrations measured in this work were obtained from the volar forearm. As it is recognized that not all skin locations are perfused to the same extent, it is reasonable to expect pharmacokinetic variation between skin sites. Future work will investigate the differences between volar forearm tissue alcohol concentrations and those obtained from the finger. Comparison of these sites is of interest, as contemporary glucose research has shown they exhibit dif-

Table 3 Tissue alcohol RMS error comparison from the elimination phase.

\begin{tabular}{lccc} 
Reference & $R M S_{\text {Exp }}=\left[\frac{\Sigma_{\left(C_{T} C_{\text {Ref }}\right)}{ }^{n}}{\mathrm{mg} / \mathrm{dL}}\right]^{1 / 2}$ & $R M S_{\text {Fit }}=\left[\frac{\Sigma_{\left(\hat{C}_{T} C_{\text {Ref }}\right)^{2}}^{n}}{\mathrm{mg} / \mathrm{dL}}\right]^{1 / 2}$ & $\begin{array}{c}R M S_{\text {Assay }}=\left(R M S_{\text {Exp }}^{2}-R M S_{\text {Fit }}^{2}\right) \\
\mathrm{mg} / \mathrm{dL}\end{array}$ \\
\hline Breath & 9.4 & 8.1 & 4.8 \\
Capillary & 11.9 & 8.8 & 8.0 \\
GC venous & 10.3 & 7.2 & 7.3 \\
\hline
\end{tabular}


ferent pharmacokinetic behavior ("the alternate site phenomenon"), with the forearm and other sites typically trailing the finger. ${ }^{27}$ Thus, there is the potential that a noninvasive tissue alcohol measurement performed on the finger could exhibit improved equilibration (larger rate constants) with blood alcohol concentration relative to forearm tissue alcohol.

Last, one of the challenges in the development of a noninvasive alcohol device is the absence of a reference value from the same compartment. While the differences between compartments have been demonstrated in a validation sense by this work, they also manifest during the calibration process. In other words, the data used to form the tissue alcohol calibration model (the regression that relates measured NIR spectra to alcohol concentrations) also contain pharmacokinetic reference error that can degrade the quality of the regression. Consequently, given that reference errors can be particularly large during the rapid changes in alcohol concentration encountered during the absorption phase of alcohol excursions, absorption measurements are typically excluded from the regression data. As the absorption phase typically accounts for approximately $1 / 3$ of the acquired clinical measurements, a means for dealing with reference error in the formation of the calibration would be advantageous.

The first-order model applied in this work offers the potential to investigate the use of a pharmacokinetic model to estimate the tissue alcohol compartment concentrations and use those values, rather than blood alcohol concentrations, in the calibration model formation. This process is somewhat circular, as the estimation of rate constants (and therefore tissue alcohol compartment estimates) requires experimental tissue alcohol concentrations, which implies that an initial regression model must be applied. Consequently, this approach could be iterative in nature, which strongly indicates the necessity of an independent validation set in order to evaluate any potential calibration benefits afforded by the estimated tissue alcohol references.

\section{Acknowledgments}

The authors thank the University of New Mexico Clinical Trial Center (UNM-CTC) for the execution of the alcohol dosing study and the clinical staff at InLight Solutions for their clinical support. We would also like to acknowledge the TriCore Forensic Toxicology Department for their analysis of venous blood samples throughout the study. This study was funded in part by a grant from the National Institute on Alcohol Abuse and Alcoholism of the U.S. National Institutes of Health.

\section{References}

1. E. W. Ciurczak, "Biomedical applications of near infrared spectroscopy," Chapter 33 in Handbook of Near-Infrared Analysis, 3rd ed., D A. Burns and E. W. Ciurczak, Eds., pp. 647-656, CRC Press, New York (2007).

2. D. Naumann, "FT-infrared and FT-raman spectroscopy in biomedical research," Appl. Spectrosc. Rev. 36(2:3), 239-298 (2001).

3. A. W. Jones, "Disposition and fate of ethanol in the body," Chapter 4 in Medical-Legal Aspects of Alcohol, 4th ed., J. C. Garriot and W. H.
Anderson, Eds., pp. 47-112, Lawyers and Judges Publishing Company, Tucson, AZ (2003).

4. BI, Inc., "Sobrieter fact sheet," www.bi.com (2002).

5. J. R. Zettl, "The determination of blood alcohol by transdermal measurement," Technical White Paper, Alcohol Monitoring Systems, Inc., Highlands Ranch, CO (2002).

6. M. D. Levitt and D. G. Levitt, "Use of a two-compartment model to predict ethanol metabolism," Alcohol Clin. Exp. Res. 24, 409-410 (2000).

7. A. Norberg, "Clinical pharmacokinetics of intravenous ethanol: relationship between the ethanol space and total body water," Thesis, Kongl Carolinska Medico Chirurgiska Institutet, 1-74 (2001).

8. A. Norberg, A. W. Jones, R. G. Hahn, and J. L. Gabrielsson, "Role of variability in explaining ethanol pharmacokinetics," Clin. Pharmacokinet 42(1), 1-31 (2003).

9. A. Norberg, J. L. Gabrielsson, A. W. Jones, and R. G. Hahn, "Within and between-subject variations in pharmacokinetic parameters of ethanol by analysis of breath, venous blood and urine," Br. J. Clin. Pharmacol. 49, 399-408 (2000).

10. G. L. Cote, "Innovative non- or minimally invasive technologies for monitoring health and nutritional status in mothers and young children," Nutrition 131, 1590S-1604S (2001).

11. H. M. Heise, A. Bittner, and R. Marbach, "Near-infrared reflectance spectroscopy for noninvasive monitoring of metabolities," Clin. Chem. Lab. Med. 38, 137-145 (2000).

12. V. V. Tuchin, Handbook of Optical Sensing of Glucose in Biological Fluids and Tissues, CRC Press, Boca Raton, FL (2008).

13. T. D. Ridder, C. D. Brown, and B. J. VerSteeg, "Framework for multivariate selectivity analysis, part II: experimental applications," Appl. Spectrosc. 59(6), 804-815 (2005).

14. T. D. Ridder, S. P. Hendee, and C. D. Brown, "Noninvasive alcohol testing using diffuse reflectance near-infrared spectroscopy," Appl. Spectrosc. 59(2), 181-189 (2005).

15. C. D. Brown and T. D. Ridder, "Framework for multivariate selectivity analysis, part I: theoretical and practical merits," Appl. Spectrosc. 59(6), 787-803 (2005).

16. R. Swift, "Transdermal measurement of alcohol consumption," Addiction 88, 1037-1039 (1993).

17. R. Swift, "Transdermal alcohol measurement for estimation of blood alcohol concentration," Alcohol Clin. Exp. Res. 24, 422-423 (2000).

18. B. T. Davies and C. K. Bowen, "Peak blood alcohol prediction: an empirical test of computer models," J. Stud. Alcohol 61, 187-191 (2000).

19. A. W. Jones, R. G. Hahn, and A. Norberg, "Concentration-time profiles of ethanol in arterial and venous blood and end-expired breath during and after intravenous infusion," J. Forensic Sci. 42, 10881094 (1997).

20. L. Lindberg, S. Brauer, P. Wollmer, L. Goldberg, A. W. Jones, and S. Olsson, "Breath alcohol concentration determined with a new analyzer using free exhalation predicts almost precisely the arterial blood alcohol concentration," Forensic Sci. Int. 168(2-3), 200-207 (2007).

21. E. Martin, M. Moll, P. Schmid, and L. Dettli, "The pharmacokinetics of alcohol in human breath, venous and arterial blood after oral ingestion," Eur. J. Clin. Pharmacol. 26, 619-626 (1984).

22. M. P. Hlastala, "The alcohol breath test-a review," Appl. Physiol. 84, 401-408 (1998).

23. A. W. Jones and L. Andersson, "Comparison of ethanol concentrations in venous blood and end-expired breath during a controlled drinking study," Forensic Sci. Int. 132, 18-25 (2003).

24. E. V. Thomas, "Adaptable multivariate calibration models for spectral applications," Anal. Chem. 72(13), 2821-2827 (2000).

25. A. W. Jones, L. Lindberg, and S. G. Olsson, "Magnitude and timecourse of arterio-venous differences in blood-alcohol concentration in healthy men," Clin. Pharmacokinet 43, 1157-1166 (2004).

26. D. G. Levitt, "The pharmacokinetics of the interstitial space in humans," BMC Clinical Pharmacol. 3(3), 1-29 (2003).

27. J. M. Ellison, J. M. Stegmann, S. L. Colner, R. H. Michael, M. K. Sharma, K. R. Ervin, and D. L. Horwitz, "Rapid changes in postprandial blood glucose produce concentration differences at finger, forearm, and thigh sampling sites," Diabetes Care 25, 961-964 (2002). 